\title{
High-risk mucosal HPV types associated with squamous cell carcinoma on the nose tip in an immunocompetent young man*
}

\author{
Camila Fátima Biancardi Gavioli ${ }^{1}$, Cyro Festa Neto ${ }^{1}$, Stephen K Tyring ${ }^{2}$, Lana Luiza da Cruz Silva ${ }^{1}$, \\ Walmar Roncalli Pereira de Oliveira ${ }^{1}$
}

DOI: http:/ / dx.doi.org/10.1590/abd1806-4841.20186919

\begin{abstract}
The oncogenic role of high-risk HPV in anogenital, head and neck, and cervical cancer is well recognized, but not in skin cancer in the general population. Some authors have demonstrated their appearance mainly on the hands and feet, particularly in the area of the nail bed, which could be due to contamination with HPV types from anogenital regions. Here, we describe a case of genital HPV associated with SCC on the nose tip in an immunocompetent young man, which was confirmed by histopathological findings and in situ hybridization. The importance of this report is to highlight the potential role of HPV in the etiology of skin cancer in an immunocompetent individual.
\end{abstract}

Keywords: Carcinoma; Carcinoma, squamous cell; Condylomata acuminata; DNA Probes, HPV; Head and neck neoplasms; Medical oncology; Papillomavirus infections; Skin neoplasms; Warts

\section{INTRODUCTION}

In the past decade, there has been a remarkable advance in our understanding of the natural history of human papillomavirus (HPV) infection and its role in the development of cervical and other anogenital cancers. Although the role of HPV in skin carcinogenesis has been well established in immunocompromised individuals, its association with cutaneous cancers in immunocompetent individuals remains a matter of much debate. ${ }^{1-4}$

HPV belongs to the Papillomaviridae family, which contains approximately 200 different HPVs types that can be found in five different genera: alpha, beta, gamma, mu, and nu. ${ }^{1,2}$ The mucosal HPV types, in which the oncogenic HPV types 16 and 18 are associated with the development of more than $70 \%$ of cervical carcinomas throughout the world, belong to the alpha genera. ${ }^{1}$ The incidence of cervical cancer is still high all over the world, despite advances in treatment as well as in prophylaxis with vaccines. ${ }^{4}$

The oncogenic role of HPV is well recognized both in cervical cancer and in squamous cell carcinoma (SCC) of the anogenital and head and neck areas, which are usually associated with high-risk HPV types such as HPVs 16, 18, 31, 33, 35, 39, 45, 51, 52, 56 and 58. . $^{1,3}$

Although the role of HPV in skin carcinogenesis has been clearly demonstrated in patients with epidermodysplasia verruciformis (EV), its association with tumor formation, specially SCC, in the general population is not well defined, as most of the studies have yielded conflicting results. ${ }^{4-8}$

Regarding the development of cutaneous tumors associated with high-risk genital HPV types in immunocompetent individuals, some authors have demonstrated their appearance mainly on the hands and feet, particularly in the area of the nail bed, which could be due to contamination with HPV types from anogenital regions. ${ }^{3}$

The presence of mucosal HPV types in skin cancers localized in different regions of the body is extremely rare in immunocompetent individuals. Here, we describe a case of genital HPV associated with SCC on the nose tip in an immunocompetent young man.

\footnotetext{
Received 26 January 2017.

Accepted 01 October 2017.

* Work conducted at the Dermatological Outpatient Clinic, Hospital das Clínicas, Faculdade de Medicina, Universidade de São Paulo, São Paulo (SP), Brazil. Financial support: None.

Conflict of interest: None.
}

1 Department of Dermatology, Faculdade de Medicina, Universidade de São Paulo, São Paulo (SP), Brazil.

Department of Dermatology, University of Texas Health Science Center, Houston, United States of America.

MaILING AdDRESS:

Camila Fátima Biancardi Gavioli

E-mail: camilinhagavioli@hotmail.com

(C)2018 by Anais Brasileiros de Dermatologia 


\section{CASE REPORT}

A 25-year-old Caucasian male, with no previous medical problems, presented with brown verrucous papules on the penis (Figure 1). He reported that the lesions had developed over the past year. Laboratory tests for syphilis and HIV infection were negative. The clinical and histopathologic diagnosis was condylomata acuminata. Hybridization in situ was performed using the GenPoint Catalyzed Signal Amplification System (DAKO, Carpentaria, CA) 16 for high-risk (HR)-HPV (types 16, 18, 31, 33, 35, 39, 45, 51, 52, 56, 58, 59, and 68; code Y1443) and low-risk (LR)-HPV (types 6 and 11) according to the manufacturer's protocols. ISH confirmed the presence of genital high-risk HPV types.

The patient was treated with $50 \%$ trichloroacetic acid and cryotherapy every 15 days during two months and topical imiqui$\bmod 5 \%$ cream on alternate days afterwards. During the treatment, the patient developed an asymptomatic ulcerated and erythematous infiltrated plaque covered by a hemorrhagic crust measuring $0.3 \mathrm{~cm}$ on the nasal tip (Figure 2).

Histopathology of the nose plaque revealed Bowenoid squamous cell carcinoma and the in situ hybridization was positive for high-risk HPV types (Figures 3-6).

\section{DISCUSSION}

Beyond the genital area, HPVs associated with cutaneous SCC are usually seen in patients with epidermodysplasia verruciformis (EV) and immunocompromised patients, e.g. organ transplant recipients (OTRs) and HIV patients. ${ }^{2-5}$ Most of these SCCs are associated with HPV related to EV (EV-HPVs). ${ }^{1,5,8}$ In immunocompetent patients, the cutaneous malignancies associated with HPV beyond the anogenital region usually occur on the periungual or subungual areas, and are associated with high-risk genital HPVs, predominantly HPV16. ${ }^{3} \mathrm{HPV}$ related to SCC localized on the nose, as we present

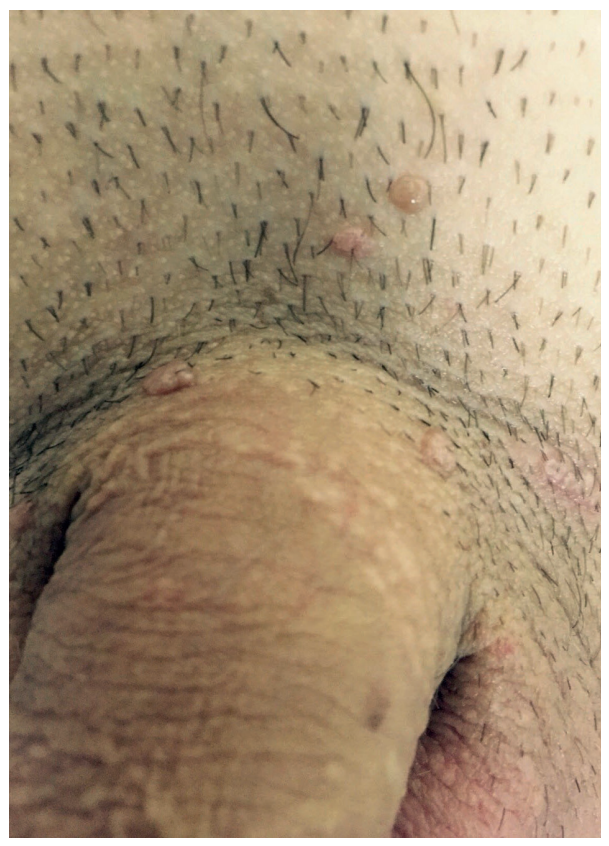

Figure 1: Brown verrucous papules on the penis

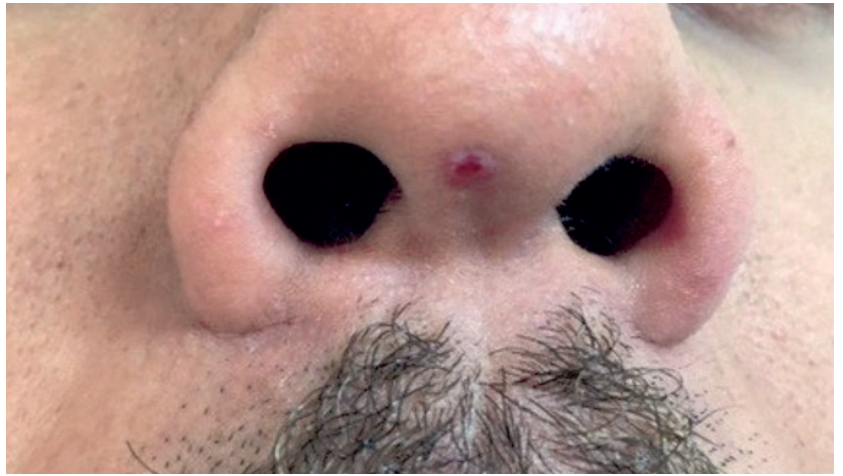

FIGURE 2: Ulcerated, infiltrated, erythematous plaque covered by a hemorrhagic crust

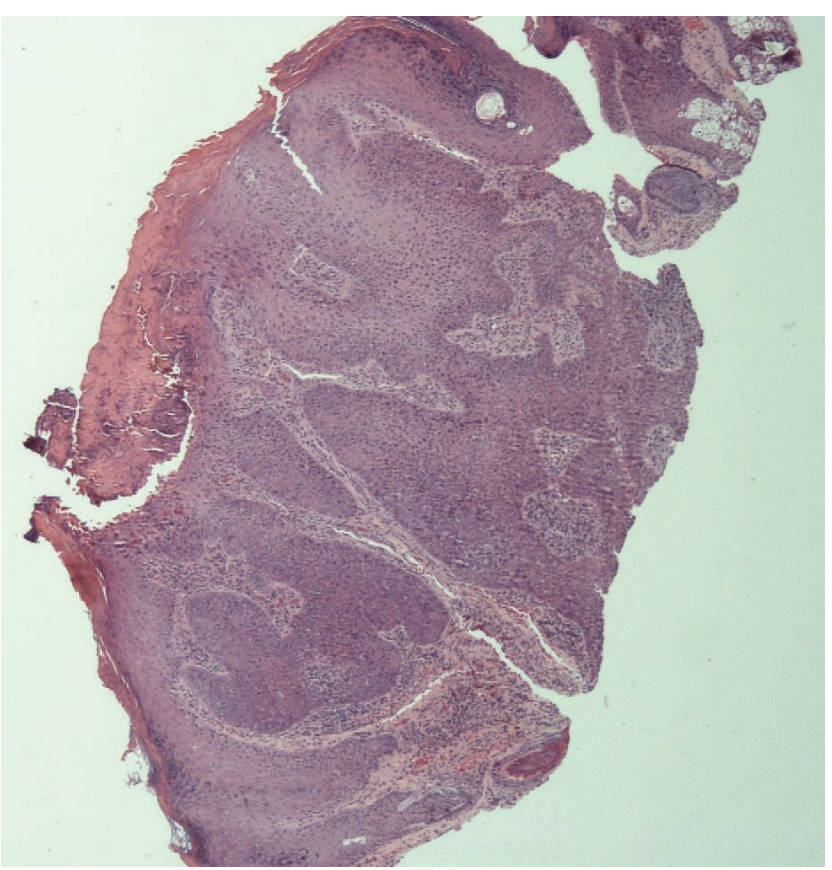

Figure 3: Bowenoid squamous cell carcinoma (Hematoxylin \& eosin, $x 40)$

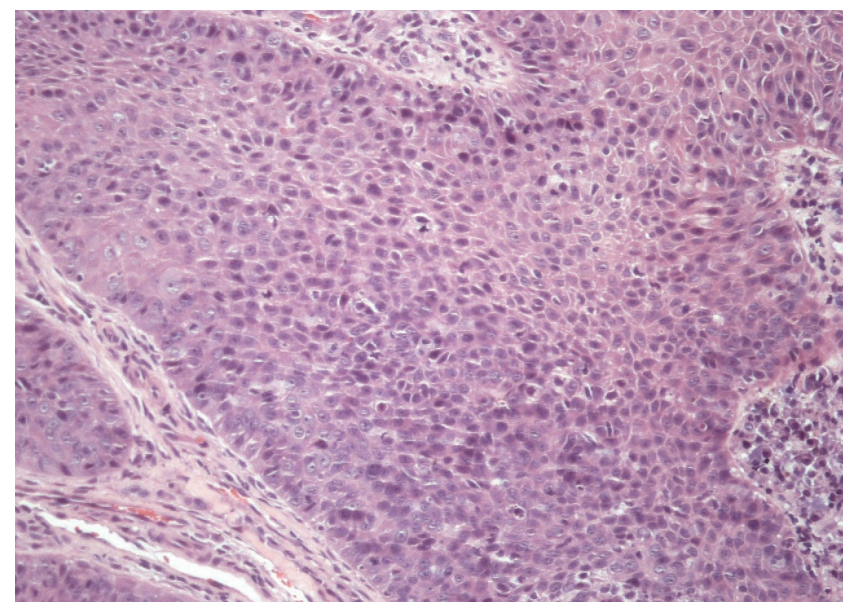

FiguRE 4: Bowenoid squamous cell carcinoma (Hematoxylin \& eosin, $x 100)$ 


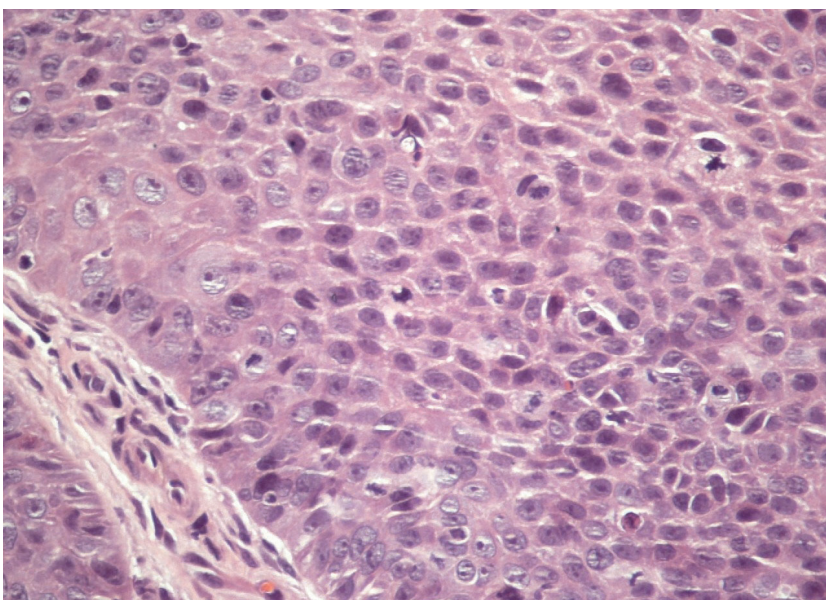

Figure 5: Bowenoid squamous cell carcinoma (Hematoxylin \& eo$\sin , x 200)$

here, appears to be extremely rare. We did not find any description in the literature of cutaneous SCC associated with oncogenic genital HPV on the nose tip in immunocompetent individuals.

High-risk HPVs are usually associated with lesions on mucosal surfaces and are the most common subtype associated with SCC of the head and neck, including oral cancers, which suggests genital-digital and genital-oral viral spread. ${ }^{3}$ This could explain the HPV transmission path from the genital area to the patient's nose.

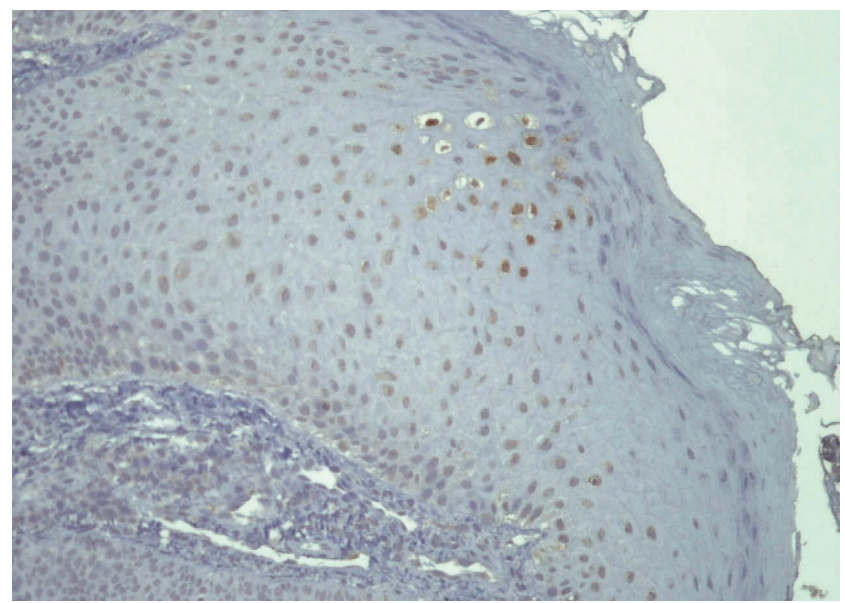

FIGURE 6: Positive in situ hybridization for high-risk HPV

Early detection of HPV induced lesions is important in order to prevent the development of cancer. The best way to diagnose an HPV infection is to confirm the presence of HPV DNA in the lesion by hybridization or PCR. The latter is expensive and is not available as a diagnostic tool in developing countries. ${ }^{9,10}$ In our case, we did not have access to PCR for HPV typing.

In summary, the importance of this report is to highlight the potential role of HPV in the etiology of skin cancer in an unexpected location in an immunocompetent individual. $\square$

7. Birch-Johansen F, Norrild B, Olesen AB, Jensen A, Kjær SK. HPV infection might play a role in the development of non-melanoma skin cancer in immunocompetent individuals. Ugeskr Laeger. 2012;174:413-7.

8. Pfister H, Fuchs PG, Majewski S, Jablonska S, Pniewska I, Malejczyk M. High prevalence of epidermodysplasia verruciformis-associated human papillomavirus DNA in actinic keratoses of the immunocompetent population. Arch Dermatol Res. 2003;295:273-9.

9. Ruhoy SM, Guinee DG, Nuovo G. Squamous cell carcinoma arising in association with verruca vulgares and HPV-2: a clinicopathologic study with p16 and p53 immunohistochemical studies and human papillomavirus in situ hybridization studies. Appl Immunohistochem Mol Morphol. 2014;22:253-61.

10. De Marchi Triglia R, Metze K, Zeferino LC, Lucci De Angelo Andrade LA. HPV in situ hybridization signal patterns as a marker for cervical intraepithelial neoplasia progression. Gynecol Oncol. 2009;112:114-8.

6. Masini C, Fuchs PG, Gabrielli F, Stark S, Sera F, Ploner M, et al. Evidence for the association of human papillomavirus infection and cutaneous squamous cell carcinoma in immunocompetent individuals. Arch Dermatol. 2003;139:890-4.

\begin{tabular}{|c|c|}
\hline \multicolumn{2}{|l|}{ AUTHORS'CONTRIBUTIONS } \\
\hline Camila Fátima Biancardi Gavioli & (iD) ORCID 0000-0002-9159-0936 \\
\hline \multicolumn{2}{|c|}{$\begin{array}{l}\text { Approval of the final version of the manuscript, Preparation and writing of the man- } \\
\text { uscript, Collecting, analysis and interpretation of data, Critical review of the literature } \\
\text { and of the manuscript }\end{array}$} \\
\hline Cyro Festa Neto & (iD) ORCID 0000-0003-3879-9981 \\
\hline \multicolumn{2}{|c|}{$\begin{array}{l}\text { Approval of the final version of the manuscript, data analysis and interpretation, Critical } \\
\text { review of the manuscript }\end{array}$} \\
\hline Stephen K Tyring & (iD) ORCID 0000-0002-7771-6571 \\
\hline
\end{tabular}

\begin{abstract}
Lana Luiza da Cruz Silva iD ORCID 0000-0003-1296-2541 Approval of the final version of the manuscript, Design and planning of the study, Preparation and writing of the manuscript, Critical review of the manuscript

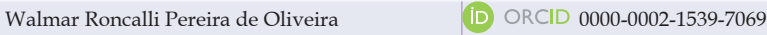
Approval of the final version of the manuscript, Design and planning of the study, Preparation and writing of the manuscript, Effective participation in research orientation, Intellectual participation in propaedeutic and/or therapeutic conduct of studied cases, data analysis and interpretation, Critical review of the literature e do $f$ the manuscript
\end{abstract}

How to cite this article: Gavioli CFB, Festa Neto C, Tyring SK, Silva LLC, Oliveira WRP. High-risk mucosal HPV types associated with squamous cell carcinoma on the nose tip in an immunocompetent young man. An Bras Dermatol. 2018;93(5):716-8. 